\title{
Risk Factors for Suicidal Ideation among Patients with Complex Regional Pain Syndrome
}

\author{
Do-Hyeong Lee', Eun Chung Noh², Yong Chul Kim³, Jae Yeon Hwang ${ }^{5}$, Sung Nyun Kim¹, \\ Joon Hwan Jang1, Min Soo Byun', and Do-Hyung Kang ${ }^{1,4 凶}$ \\ 1'Department of Neuropsychiatry, Seoul National University Hospital, Seoul, Republic of Korea \\ ${ }^{2}$ Interdisciplinary Program of Neuroscience, Seoul National University, Seoul, Republic of Korea \\ ${ }^{3}$ Department of Anesthesiology and Pain Medicine, Seoul National University Hospital, Seoul, Republic of Korea \\ ${ }^{4}$ Department of Psychiatry, Seoul National University College of Medicine, Seoul, Republic of Korea \\ ${ }^{5}$ Department of Psychiatry, SMG-SNU Boramae Medical Center, Seoul, Republic of Korea
}

Objective Chronic pain frequently coexists with psychiatric symptoms in patients diagnosed with complex regional pain syndrome (CRPS). Previous studies have shown a relationship between CRPS and the risk of suicide. The purpose of this study was to assess risk factors for suicidal ideation in patients with CRPS.

Methods Based on criteria established by the International Association for the Study of Pain, 39 patients diagnosed with CRPS Type 1 or Type 2 were enrolled in this study. Suicidal ideation was assessed using item 3 of the Hamilton Depression Rating Scale (HAMD), and symptoms of pain were evaluated using the short form of the McGill Pain Questionnaire (SF-MPQ). Psychiatric symptoms were assessed in using the Structured Clinical Interview for DSM-IV Disorders (SCID-I, SCID-II), the HAMD, the Hamilton Anxiety Rating Scale (HAMA), the Global Assessment of Functioning Scale (GAF), and the Pittsburgh Sleep Quality Index (PSQI).

Results Twenty-nine patients (74.4\%) were at high risk and 10 (25.6\%) were at low risk for suicidal ideation. Risk factors significantly associated with suicidal ideation included depression $(\mathrm{p}=0.002)$, severity of pain $(\mathrm{p}=0.024)$, and low scores on the $\mathrm{GAF}$ ( $\mathrm{p}=0.027)$. No significant correlations were found between suicidal ideation and anxiety or quality of sleep.

Conclusion Significant risk factors for suicidal ideation in patients with CRPS include severity of pain, depressive symptoms, and decreased functioning. These results suggest that psychiatric evaluation and intervention should be included in the treatment of CRPS.

Psychiatry Investig 2014;11:32-38

Key Words Complex regional pain syndrome, Depression, Anxiety, Suicidal ideation.

\section{INTRODUCTION}

Pain is associated with higher risk of suicide. ${ }^{1-3}$ In a recent review, Tong et al. ${ }^{1}$ reported that the lifetime prevalence of suicidal ideation (SI) and suicide attempts for patients with chronic pain were $5-14 \%$ and $20 \%$, respectively. Edwards et al. ${ }^{3}$ found that more than $30 \%$ of subjects suffering from chronic pain reported some form of recent suicidal ideation. Large population-based studies have shown an association between

Received: May 12, 2013 Revised: August 12, 2013

Accepted: August 16, 2013 Available online: October 16, 2013

$\triangle$ Correspondence: Do-Hyung Kang, MD, PhD

Department of Psychiatry, Seoul National University College of Medicine, 101 Daehak-ro, Jongno-gu, Seoul 110-744, Republic of Korea

Tel: +82-2-2072-0690, Fax: +82-2-744-7241, E-mail: basuare@paran.com

(a) This is an Open Access article distributed under the terms of the Creative Commons Attribution Non-Commercial License (http://creativecommons.org/licenses/bync/3.0) which permits unrestricted non-commercial use, distribution, and reproduction in any medium, provided the original work is properly cited. various types of chronic pain conditions (e.g., migraine headaches, back pain, arthritis, fibromyalgia) and suicide risk, even with adjustment for mental disorders. ${ }^{4}$

Although it may be difficult to determine whether a mental disorder is a preexisting condition or is subsequent to chronic pain, comorbidity significantly increases the risk of suicide. ${ }^{2}$ Findings have shown that depression is the most common comorbid symptom, and studies indicate that $31-100 \%$ of patients with chronic pain suffer from depression. ${ }^{5}$ Moreover, Kroenke et al. ${ }^{6}$ reported that changes in the severity of pain predicted changes in the severity of depressive symptoms experienced by patients with chronic pain and vice versa. Symptoms of anxiety are similarly common in the medical profiles of patients with chronic pain. In a population-based study, the prevalence of anxiety disorders was 35\% in persons with chronic pain compared with $18 \%$ in the general population. ${ }^{7}$ However, it is important to note that these studies used data 
collected primarily from patients with musculoskeletal and other relatively common neuropathic pain-related conditions (i.e., patients with pain associated with migraine headaches, arthritis, back pain, fibromyalgia, etc.).

Complex regional pain syndrome (CRPS) is a medical condition whose primary symptom is chronic distressing pain and it has been subdivided into type I (reflex sympathetic dystrophy) and type II (causalgia). ${ }^{8}$ CRPS may involve pain that is localized to an extremity (the arm, hand, leg, or foot) or pain that affects multiple parts of the body. Localized pain affecting a limb is often accompanied by impairment in the functioning of that extremity and the possibility of ongoing pain and severe disability. ${ }^{9}$ Although the mechanism underpinning CRPS remains unknown, investigators have proposed various hypotheses, including that CRPS is a systemic disease involving the central nervous system (CNS) and the peripheral nervous system (i.e., neuropathic) and associated interactions between the immune system and sensitive nociceptive nervous system transmission. $^{10,11}$

Although CRPS has properties similar to those of other conditions involving peripheral neuropathy, it also has several distinct characteristics. For example, the subjective experience of pain in CRPS is accompanied by sudomotor and vasomotor dysfunction (i.e., changes in skin color and/or temperature). ${ }^{12}$ CRPS is viewed as among the most painful of all known diseases, with patients' scores on the McGill Pain Questionnaire (mean MPQ score of 42 of a possible 50) being among the highest of any diagnostic group, surpassing even the scores of patients who have experienced amputation of a limb or childbirth. ${ }^{13,14}$

It is prudent for clinicians to include a thorough suicide risk assessment in medical examinations of patients with CRPS given their apparent vulnerability due to functional impairments and severe pain and to the possibility of psychiatric symptoms associated with depression and other psychological diagnoses. Lohnberg's review ${ }^{12}$ of the relationship between CRPS and psychological and psychosocial factors found that CRPS was associated with depression, anxiety, reduced quality of life, and impaired occupational functioning. Additionally, a web-based epidemiological survey with CRPS-1 patients estimated that $20 \%$ had attempted suicide, and $46.4 \%$ reported experiencing suicidal ideation. ${ }^{15}$ However, few data about psychological comorbidity with respect to suicide risk factors in CRPS patients are available.

The primary aim of this study was to identify clinical factors related to suicidal ideation in patients with CRPS. Secondarily, we evaluated psychiatric comorbidity in this population. Results indicated an association between suicidal ideation and various psychiatric symptoms. We hypothesized that lifetime comorbid psychiatric symptomatology would increase the risk for suicidal ideation among individuals with CRPS.

\section{METHODS}

\section{Participants}

The subjects were outpatients at the Pain Clinic of Seoul National University Hospital. Patients included in the study met the following criteria: 1) aged 18 years or older; 2) presentation with CRPS symptoms; and 3) voluntary provision of informed consent for participation in the study. Exclusion criteria consisted of 1) younger than 18 years of age, 2) fewer than 6 months of CRPS treatment, 3) a medical history of neurological disease, 4) a history of substance abuse, and 5) previous treatment for any type of neuropsychiatric condition.

After obtaining approval to conduct the study from the Institutional Review Board, the procedures used in the study were fully explained to all subjects, who then provided informed consent to participate. The patient subjects were evaluated by physicians to confirm the presence of CRPS as defined by the International Association for the Study of Pain (IASP). Each subject was subsequently assessed by neuropsychiatric specialists who were blind to each patient's clinical profile to determine the presence and nature of any psychiatric symptoms.

\section{Measurements}

\section{Structured Clinical Interview for the DSM IV-I, II}

The Structured Clinical Interview for DSM-IV Disorders (SCID) is a diagnostic tool used to identify DSM-IV Axis $\mathrm{I}^{16}$ and Axis II disorders (major mental disorders and personality disorders, respectively). ${ }^{17}$ The instrument was designed to be administered by a clinician or trained mental health professional. The Korean version was used in this study.

\section{Psychiatric symptoms}

Psychiatric characteristics were assessed with Hamilton Rating Scale for Depression (HAMD) ${ }^{18}$ and the Hamilton Rating Scale for Anxiety (HAMA). ${ }^{19}$ The HAMD is a multiple-choice questionnaire that clinicians use to rate the severity of a patient's depression. A score of $0-7$ is considered to be normal, and scores of 20 or more indicate moderately severe depression. For a comparison of depressive symptoms, we summed the HAMD items with the exception of item 3 (the suiciderisk item), which we excluded to avoid biasing the relationship between depression severity and suicide risk. The Hamilton Anxiety Rating Scale (HAMA) is a questionnaire used by clinicians to rate the severity of a patient's anxiety. It contains 14 symptom-oriented questions. Each of these symptoms is given a severity rating ranging from not present (0) to very severe (4). Total scores of $0-17$ are considered to indicate mild anxiety, 
those 18-25 mild to moderate anxiety, and those 26-30 moderate to severe anxiety. Subjects were also rated on the Global Assessment of Functioning (GAF) Scale, which is based on Axis V of the Diagnostic and Statistical Manual of Mental Disorders, ${ }^{20}$ to assess psychological, social, and occupational functioning. Scores range from 1-100, with higher scores reflecting better functioning.

\section{Suicidal ideation}

The low-suicidal ideation (SI) group included those with scores of 1 or below on HAMD item 3 ( 0 =suicide ideation absent, $1=$ feels that life is worth living), and the high-SI group included those with HAMD item 3 scores above 1 (2=wishes he were dead or any thoughts of possible death to self; $3=$ suicidal ideas or gesture; $4=$ attempts at suicide). This cutoff value has been commonly used in previous studies. ${ }^{21-23}$

\section{Pittsburgh Sleep Quality Index}

The PSQI ${ }^{24}$ is a self-administered questionnaire that assesses subjective sleep quality during the previous month. The selfrated items on the PSQI generate seven component scores (range of subscale scores, 0-3): sleep quality, sleep latency, sleep duration, habitual sleep efficiency, sleep disturbance, use of sleeping medication, and daytime dysfunction. The sum of these seven component scores yields one global score for subjective sleep quality (range, 0-21); higher scores represent po-orer subjective sleep quality, and those $>5$ are associated with poor sleep quality.

\section{Short form of the McGill Pain Questionnaire}

The short form of the McGill Pain Questionnaire (MPQ$\mathrm{SF})^{25}$ was used as an index of pain severity. Five scores are derived from the SF-MPQ; the Sensory Pain Rating Index (SPRI), the Affective Pain Rating Index (A-PRI), the Total Pain Rating Index (T-PRI), the Present Pain Intensity-Visual Analogue Scale (PPI-MPQ-VAS), and the overall intensity of total pain. In this study, a visual analogue scale (MPQ-VAS) was used to assess pain severity. MPQ-VAS is a visual analogue scale that uses a $10-\mathrm{cm}$ line divided into $1-\mathrm{cm}$ sections. $\mathrm{Pa}$ tients indicate their level of pain by marking the appropriate place on the scale from 0 (no pain) to 10 (the worst possible pain). ${ }^{26}$

\section{Statistical analysis}

Between-group comparisons involving categorical data were performed using the chi-square statistic corrected for continuity; between-group comparisons involving continuous data (age and socio-economic status) were calculated using Student's t-test. Pearson's correlation analysis was used to assess correlations between suicidal ideation and other variables.

\section{RESULTS}

\section{Demographic variables}

The sample included 39 patients with CRPS; 33 (84.6\%) were diagnosed with CRPS Type 1, and six (15.4\%) were diagnosed with CRPS Type II. Males accounted for $61.5 \%(n=24)$ of the patients, and females $38.5 \%(n=15)$. The mean age of the patients at their initial evaluation at the pain clinic was 37.87 $( \pm 11.94)$ years, and the mean number of years of education was $12.49( \pm 2.68)$ years. Patients reported having CRPS symptoms for a mean of 2.84 years $( \pm 2.49)$ at the time of evaluation and had been prescribed 4.28 (range, $0-7$ ) different kinds of medications on average. The most frequently used medications were anticonvulsants and antidepressants. We found no significant differences between the two groups in terms of demographic characteristics and medications (Table 1). The most common psychiatric comorbidity according to the SCID was depression. Among those with depression, 19 (48.7\%) met the criteria for major depressive disorder, and 6 patients (15.4\%) were diagnosed with depressive disorder NOS. Two patients (5.1\%) met the criteria for bipolar II disorder, and one patient met the criteria for panic disorder. In terms of Axis II, seven patients (17.9\%) met the criteria for obsessive-compulsive personality disorder, and one each met the criteria for borderline personality disorder, avoidant personality disorder, and narcissistic personality disorder. Eight (18.2\%) subjects were not diagnosed with any comorbid psychiatric disorders by the SCID-I or II. No significant differences were found between the two groups in SCID results (Table 2).

\section{Clinical characteristics of patients with CRPS: low and moderate-to-high SI}

Figure 1 presents the differences between low-SI and highSI groups in terms of psychiatric symptoms and other variables. Twenty-nine patients (74.4\%) were placed in the high-SI group, and 10 (25.6\%) were placed in the low-SI group.

Anxiety was not a significant predictor of suicide risk, but depressive symptoms were. The HAMD average score of highSI group (25.00 $\pm 6.94 \mathrm{SD})$ was significantly higher than that of the low-SI group $(14.03 \pm 9.49 ; \mathrm{F}=1.67, \mathrm{t}=-3.35, \mathrm{df}=37$, $\mathrm{p}=0.002$ ).

The average pain intensity for each year after symptom onset was $5.50(\mathrm{SD}=2.3)$. The high-SI group reported greater pain intensity $(6.90 \pm 2.81)$ than did the low-SI group $(5.02 \pm 1.93 ; \mathrm{t}=-2.36, \mathrm{df}=37, \mathrm{p}=0.02)$. The low-SI group also had significantly higher GAF scores $(53.38 \pm 14.56)$ than did the high-SI group (44.90 $\pm 7.68 ; \mathrm{t}=2.33, \mathrm{df}=30.2, \mathrm{p}=0.03)$. No differences were found with regard to sleep quality, but the majority of patients reported poor sleep quality (13.64 \pm 4.22$)$. Significant correlations were found between suicidal ideation 
Table 1. Demographic and clinical characteristics of patients

\begin{tabular}{|c|c|c|c|c|c|c|}
\hline & Total $(\mathrm{N}=39)$ & Low SI (N=29) & High SI $(\mathrm{N}=10)$ & $\mathrm{T}, \chi^{2}$ & $\mathrm{df}$ & $\mathrm{p}$ \\
\hline Age (years) & $37.87 \pm 11.97$ & $36.07 \pm 11.90$ & $43.10 \pm 11.12$ & -1.64 & 37 & $0.11^{*}$ \\
\hline Education (years) & $12.49 \pm 2.68$ & $12.45 \pm 2.92$ & $12.60 \pm 1.96$ & -0.15 & 37 & $0.88^{*}$ \\
\hline Duration (years) & $2.84 \pm 2.49$ & $2.60 \pm 2.23$ & $3.54 \pm 3.13$ & -1.03 & 37 & $0.31^{*}$ \\
\hline \multicolumn{7}{|l|}{ Gender (\%) } \\
\hline M & $24(61.5 \%)$ & 19 & 5 & 0.76 & 1 & $0.38^{\dagger}$ \\
\hline $\mathrm{F}$ & $15(38.5 \%)$ & 10 & 5 & & & \\
\hline \multicolumn{7}{|l|}{ Diagnosis } \\
\hline CRPS I & $33(84.6 \%)$ & $25(86.2 \%)$ & $8(80 \%)$ & 0.22 & 1 & $0.64^{\dagger}$ \\
\hline CRPS II & $6(15.4 \%)$ & $4(13.8 \%)$ & $2(20 \%)$ & & & \\
\hline \multicolumn{7}{|l|}{ Medications (present) } \\
\hline Antidepressants & 33 & $24(82.8 \%)$ & $9(90.0 \%)$ & 0.30 & 1 & $0.58^{\dagger}$ \\
\hline Opiates & 17 & $12(41.4 \%)$ & $5(50.0 \%)$ & 0.23 & 1 & $0.64^{\dagger}$ \\
\hline Antipsychotics & 10 & $6(20.7 \%)$ & $4(40.0 \%)$ & 1.45 & 1 & $0.23^{\dagger}$ \\
\hline Anticonvulsants & 33 & $24(82.8 \%)$ & $9(90.0 \%)$ & 0.30 & 1 & $0.58^{\dagger}$ \\
\hline
\end{tabular}

Continuous variables: Mean \pm SD. *independent sampled t-test, ${ }^{\dagger}$ chi-square test. SD: standard deviation, CRPS: Complex Regional Pain Syndrome, SI: suicidal ideation

Table 2. Psychiatric co-morbidities assessed by SCID I and II

\begin{tabular}{|c|c|c|c|c|c|c|}
\hline SCID & Total $(\mathrm{N}=39)$ & Low SI (N=29) & High SI $(\mathrm{N}=10)$ & $\chi^{2}$ & df & $\mathrm{p}$ \\
\hline \multicolumn{7}{|l|}{ Axis I } \\
\hline None & $11(28.2 \%)$ & 10 & 1 & 8.33 & 4 & $0.08^{\dagger}$ \\
\hline Bipolar disorder & $2(5.1 \%)$ & 0 & 2 & & & \\
\hline Major depression & $19(48.7 \%)$ & 13 & 6 & & & \\
\hline Depressive NOS & $6(15.4 \%)$ & 5 & 1 & & & \\
\hline Panic disorder & $1(2.6 \%)$ & 1 & 0 & & & \\
\hline \multicolumn{7}{|l|}{ Axis II } \\
\hline None & $29(74.4 \%)$ & 24 & 5 & 8.31 & 4 & $0.08^{\dagger}$ \\
\hline Obsessive compulsive PD & $7(17.9 \%)$ & 3 & 4 & & & \\
\hline Borderline PD & $1(2.6 \%)$ & 1 & 0 & & & \\
\hline Avoidant PD & $1(2.6 \%)$ & 0 & 1 & & & \\
\hline Narcissistic PD & $1(2.6 \%)$ & 1 & 0 & & & \\
\hline
\end{tabular}

${ }^{\dagger}$ chi-square test. SCID: Structured Clinical Interview for DSM-IV, PD: personality disorder, SI: suicidal ideation, NOS: not otherwise specified

and HAMD ( $\mathrm{r}=0.72, \mathrm{p}=0.00)$, HAMA $(\mathrm{r}=0.58, \mathrm{p}=0.00)$, PSQI $(\mathrm{r}=0.43, \mathrm{p}<0.01)$, and GAF $(\mathrm{r}=-0.56, \mathrm{p}=0.00)$ scores.

\section{DISCUSSION}

This cross-sectional study gathered data about patients with CRPS who had no previous psychiatric symptoms using neuropsychiatric scales administered by a psychiatric specialist at a tertiary university-based pain center. To the best of our knowledge, this is the first study to assess predictors of suicide risk in patients with CRPS. The strengths of this study include its use of a structured interview (SCID) to diagnose mental disorders.

In terms of mental health-related variables, although we found no significant differences in the SCID results for the two groups, the HAMD scores were significantly higher in the high-SI than in the low-SI group, and we found a positive correlation between suicidal ideation and depressive symptoms. Little research has assessed the causal relationship between suicidal ideation and depression in patients with CRPS, but several studies on chronic pain have revealed that the presence of pain may hinder the detection and treatment of depression, and patients in pain are in a high risk for suicide..$^{27-29}$ Surveys of patients with fibromyalgia have reported that depres- 


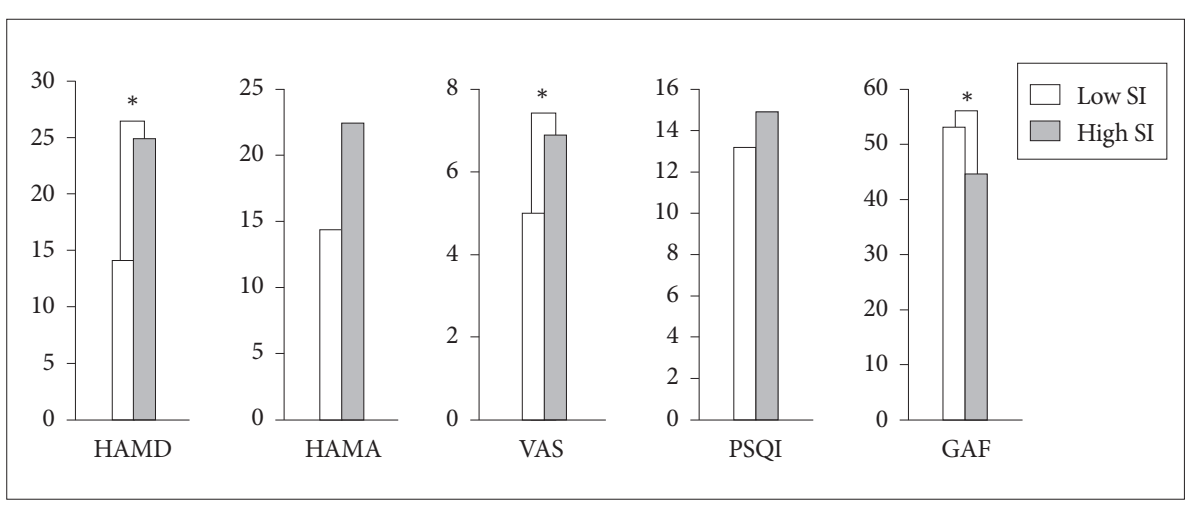

Figure 1. Comparison of symptoms profiles for patients with CRPS between high SI and low SI. ${ }^{*} p<0.05$. HAMD: Korean version of the Hamilton Depression Rating Scale, HAMA: Korean version of the Hamilton Anxiety Rating Scale, VAS (MPQ-SF): Visual Analogue Scale, PSQI (Total): Pittsburgh Sleep Quality Index, GAF: Global Assessment of Functioning, SI: suicidal ideation. sion is positively correlated with suicide risk ${ }^{30}$ and that depressed chronic pain patients who were experiencing suicidal ideation were twice as likely compared with non-depressed patients to have attempted suicide at least once before the study. ${ }^{1,31}$ Additionally, Edwards et al. ${ }^{3}$ reported that depression was the most consistent predictor of the presence and degree of suicidal ideation in patients with chronic pain. This result suggests that the significant risk factor is not the diagnosis of depression per se but the severity of depressive symptoms and their aggravation at a particular point in time. In our study, no differences were found between the groups group in SCID results; only depressive symptoms were significantly different. Therefore, clinicians should consider performing or ordering a psychiatric evaluation when patients with CRPS show depressive symptoms or suicidal ideation.

Pain severity strongly distinguished the high-SI from the low-SI group and was significantly correlated with suicidal ideation. Previous studies have suggested that persistent pain is associated with elevated rates of suicide-related behaviors. Ratcliffe et al. ${ }^{2}$ reported that the presence of chronic pain was uniquely associated with suicidal ideation and suicidal attempts after adjusting for common mental disorders and comorbidity, and several studies have reported a positive relationship between pain severity and suicidal ideation. ${ }^{3,32}$ These results suggest the importance of assessing suicide risk in patients with painful conditions, even in the absence of comorbid mental disorders.

We did not control for the interaction between pain and depression in our analysis of the predictive value of the factors under examination. However, several studies have shown a causal association between depression and pain in patients with CRPS. Kroenke et al. ${ }^{6}$ showed that pain and depression have strong and similar effects on each another over the course of 12 months and suggested a bidirectional and potentially causal relationship between pain and depression. Additionally, McDaniel ${ }^{33}$ reported three cases in which electroconvulsive treatment (ECT) was administered to patients with CRPS and depressive mood, and the results showed that the treatments improved both the depression and the pain symptoms. Considering that the severity of CRPS is the highest level among pain disorders, ${ }^{34}$ patient with CRPS would show significantly higher level of depression. Our results indicate that depression is the most common psychological symptom among those with CPRS. Approximately $70 \%$ of patients with this condition qualified for a diagnosis of depressive disorder according to the SCID, and more than $80 \%$ patients had been treated with antidepressants. We could not identify whether the treatment of depressive disorders led directly to improvement in pain symptoms, but it has been reported that pain severity is significantly correlated with depressive mood and that these are both associated with suicide risk. ${ }^{27,31,35}$ Therefore it is important to assess comorbid depressive symptoms starting at the first visit to prevent worsening of pain symptoms and suicidal ideation.

No difference was found between the high- and low-SI groups in terms of anxiety. This result is similar to those of other studies that reported that symptoms of anxiety were relatively less significant predictors of SI severity compared with depression. ${ }^{3}$ However, as with depressive symptoms, several studies have suggested a relationship between anxiety and pain. ${ }^{36,37}$ One meta-analysis showed an association between anxiety sensitivity and fearful appraisals of pain in clinical and nonclinical samples. ${ }^{38}$ Additionally, Means-Christensen and colleagues found an association between pain symptoms and both depression and anxiety. ${ }^{39}$ In our study, patients with CRPS showed moderate levels of anxiety (HAMA: $16.38 \pm 12.12$ ), and a moderate-to-large effect size was found for its association with pain ( $\mathrm{r}=0.58, \mathrm{p}=0.00)$. Although we did not find significant results in this regard, these studies suggest that elevated levels of anxiety are associated with the severity of pain, which may be associated with subsequent depression and suicide risk. ${ }^{30}$

The two SI groups differed significantly in their scores on the GAF, which assesses patients' 1-year psychological, social, and occupational functioning and has been accepted as stronger predictor psychiatric symptoms. ${ }^{40,41}$ Additionally, this mea- 
sure has been used to evaluate the functioning of patients with chronic and acute pain. ${ }^{42-44}$ This finding suggests that patients with CRPS have significantly higher rates of maladaptive functioning, which may relate to their increased psychopathology and lower general functioning, as evidenced by their increased HAMD scores and their lower GAF scores, respectively.

Although we found no significant difference with regard to suicidal ideation, most patients (97\%) reported higher than 5 in PSQI total score (mean $\pm \mathrm{SD}=13.64 \pm 4.22$ ) which means poor quality of sleep and we found a significant correlation between suicidal ideation and PSQI total scores $(r=0.28$, $\mathrm{p}=0.00$ ). Several previous surveys about chronic pain suggested that various etiologies significantly interfere with sleep..$^{30,45}$ Most of the patients suffered from sleep difficulties after they began experiencing chronic pain, and several studies showed a positive correlation between pain intensity and degree of sleep disturbance. ${ }^{46}$ Bigatti et al. ${ }^{45}$ suggested that improving the sleep quality of fibromyalgia patients would decrease their pain and depression. These results probably do not differ from those of other pain patients, but few studies have focused on patients with CRPS only. Thus, a large multicenter prospective study is needed to validate this association between sleep quality and pain, especially in patients with CRPS.

Similar with other chronic pain conditions, biological, psychological, and social factors are important in treatment of CRPS. Previous studies reported that chronic pain brings about disability, emotional arousal which in turn aggravates pain severity. ${ }^{47}$ Various therapies such as cognitive behavioral therapy, ergonomic training physiotherapy, patient education and medication have been suggested. ${ }^{48,49}$ These psychosocial and behavioral intervention as part of multidisciplinary treatment approach also would be effective in improvement of symptoms for CRPS.

Several limitations of this study should be considered when interpreting the findings. First, because the present study is cross-sectional, these results may not be generalizable to all CRPS patients. Although longitudinal investigations are necessary to replicate these findings, this study should assist patients with CRPS who may be at risk for suicidal ideation by underscoring the importance of regular assessment and psychological treatment. Second, the sample size was relatively small, which may have resulted in a selection bias. Several epidemiologic studies have reported a female to male ratio ranging from $2: 1$ to $4: 1^{50,51}$; however, the female to male ratio in our data was 1:1.6. This may be due to the low estimated incidence of CRPS (5.46-26.2 per 100,000 person-years) $)^{50,52}$ and to the fact that this study was conducted at a single center, which made it difficult to recruit an adequate number of patients over a short period of time. A large multicenter research needs to be per- formed to validate these results. Third, several modalities identified as important treatments for pain symptoms, such as ketamine infusion and nerve blocks, were not controlled in the present study.

CRPS is recognized as one of the most difficult conditions to treat among pain syndromes. This study found that the factors most strongly associated with suicide in patients with CRPS were depression, pain severity, and general functioning. Taken together, these results should reinforce the importance of psychiatric evaluations and multidisciplinary approach in the treatment of patients with CRPS.

\section{Acknowledgments}

This study was supported by the Original Technology Research Program for Brain Science through the National Research Foundation of Korea (NRF) funded by the Ministry of Education, Science and Technology (2012-0006587).

\section{REFERENCES}

1. Tang NK, Crane C. Suicidality in chronic pain: a review of the prevalence, risk factors and psychological links. Psychol Med 2006;36:575586.

2. Ratcliffe GE, Enns MW, Belik SL, Sareen J. Chronic pain conditions and suicidal ideation and suicide attempts: an epidemiologic perspective. Clin J Pain 2008;24:204-210.

3. Edwards RR, Smith MT, Kudel I, Haythornthwaite J. Pain-related catastrophizing as a risk factor for suicidal ideation in chronic pain. Pain 2006;126:272-279.

4. Ilgen MA, Zivin K, McCammon RJ, Valenstein M. Pain and suicidal thoughts, plans and attempts in the United States. Gen Hosp Psychiatry 2008;30:521-527.

5. Romano JM, Turner JA. Chronic pain and depression: does the evidence support a relationship? Psychol Bull 1985;97:18-34.

6. Kroenke K, Wu J, Bair MJ, Krebs EE, Damush TM, Tu W. Reciprocal relationship between pain and depression: a 12-month longitudinal analysis in primary care. J Pain 2011;12:964-973.

7. McWilliams LA, Cox BJ, Enns MW. Mood and anxiety disorders associated with chronic pain: an examination in a nationally representative sample. Pain 2003;106:127-133.

8. Stanton-Hicks M. Complex regional pain syndrome (type I, RSD; type II, causalgia): controversies. Clin J Pain 2000;16(2 Suppl):S33-S40.

9. Galer BS, Henderson J, Perander J, Jensen MP. Course of symptoms and quality of life measurement in Complex Regional Pain Syndrome: a pilot survey. J Pain Symptom Manage 2000;20:286-292.

10. Üçeyler N, Sommer C. Status of immune mediators in painful neuropathies. Curr Pain Headache Rep 2008;12:159-164.

11. Marchand F, Perretti M, McMahon SB. Role of the immune system in chronic pain. Nat Rev Neurosci 2005;6:521-532.

12. Lohnberg JA, Altmaier EM. A review of psychosocial factors in complex regional pain syndrome. J Clin Psychol Med Settings 2013;20:247254.

13. Tahmoush AJ. Causalgia: redefinition as a clinical pain syndrome. Pain 1981;10:187-197.

14. Melzack R, Wall PD, Ty TC. Acute pain in an emergency clinic: latency of onset and descriptor patterns related to different injuries. Pain 1982; 14:33-43.

15. Agarwal S, Broatch J, Raja S. Web-based epidemiological survey of complex regional pain syndrome-1. Anesthesiology 2005;103:A902.

16. First MB, Gibbon M. User's Guide for the Structured Clinical Interview for DSM-IV Axis I Disorders: SCID-1 Clinician Version. Wash- 
ington, DC: American Psychiatric Association; 1997.

17. First MB, Gibbon M. User's Guide for the Structured Clinical Interview for DSM-IV Axis II Personality Disorders: SCID-II. Washington, DC: American Psychiatric Association; 1997.

18. Hamilton M. A rating scale for depression. J Neurol Neurosurg Psychiatry 1960;23:56-62.

19. Hamilton M. The assessment of anxiety states by rating. Br J Med Psychol 1959;32:50-55.

20. American Psychiatric Association. Diagnostic Criteria from DSM-IV. Washington, DC: American Psychiatric Association Inc; 1994.

21. Darbinyan V, Aslanyan G, Amroyan E, Gabrielyan E, Malmström C, Panossian A. Clinical trial of Rhodiola rosea L. extract SHR-5 in the treatment of mild to moderate depression. Nord J Psychiatry 2007;61: 343-348.

22. Beasley CM Jr, Ball SG, Nilsson ME, Polzer J, Tauscher-Wisniewski S, Plewes J, et al. Fluoxetine and adult suicidality revisited: an updated meta-analysis using expanded data sources from placebo-controlled trials. J Clin Psychopharmacol 2007;27:682-686.

23. Perlis RH, Beasley CM Jr, Wines JD Jr, Tamura RN, Cusin C, Shear D, et al. Treatment-associated suicidal ideation and adverse effects in an open, multicenter trial of fluoxetine for major depressive episodes. Psychother Psychosom 2007;76:40-46.

24. Buysse DJ, Reynolds CF 3rd, Monk TH, Berman SR, Kupfer DJ. The Pittsburgh Sleep Quality Index: a new instrument for psychiatric practice and research. Psychiatry Res 1989;28:193-213.

25. Melzack R. The short-form McGill Pain Questionnaire. Pain 1987;30: 191-197.

26. Mason VL, Skevington SM, Osborn M. Assessing the properties of the WHOQOL-Pain: quality of life of chronic low back pain patients during treatment. Clin J Pain 2010;26:583-592.

27. Cheatle MD. Depression, chronic pain, and suicide by overdose: on the edge. Pain Med 2011;12(Suppl 2):S43-S48.

28. Kroenke K, Price RK. Symptoms in the community. Prevalence, classification, and psychiatric comorbidity. Arch Intern Med 1993;153:24742480.

29. Magni G, Marchetti M, Moreschi C, Merskey H, Luchini SR. Chronic musculoskeletal pain and depressive symptoms in the National Health and Nutrition Examination I. Epidemiologic follow-up study. Pain 1993;53:163-168.

30. Calandre EP, Vilchez JS, Molina-Barea R, Tovar MI, Garcia-Leiva JM, Hidalgo J, et al. Suicide attempts and risk of suicide in patients with fibromyalgia: a survey in Spanish patients. Rheumatology (Oxford) 2011;50:1889-1893.

31. Fisher BJ, Haythornthwaite JA, Heinberg LJ, Clark M, Reed J. Suicidal intent in patients with chronic pain. Pain 2001;89:199-206.

32. Hinkley BS, Jaremko ME. Effects of pain duration on psychosocial adjustment in orthopedic patients: the importance of early diagnosis and treatment of pain. J Pain Symptom Manage 1994;9:175-185.

33. McDaniel WW. Electroconvulsive therapy in complex regional pain syndromes. J ECT 2003;19:226-229.
34. Wall PD, Melzack R, Bonica JJ. Textbook of Pain. London, UK: Churchill Livingstone; 1999.

35. Bair MJ, Robinson RL, Eckert GJ, Stang PE, Croghan TW, Kroenke K. Impact of pain on depression treatment response in primary care. Psychosom Med 2004;66:17-22.

36. Velikova G, Selby PJ, Snaith PR, Kirby PG. The relationship of cancer pain to anxiety. Psychother Psychosom 1995;63:181-184.

37. Bair MJ, Poleshuck EL, Wu J, Krebs EK, Damush TM, Tu W, et al. Anxiety but not social stressors predict 12-month depression and pain severity. Clin J Pain 2013;29:95-101.

38. Ocañez KL, McHugh RK, Otto MW. A meta-analytic review of the association between anxiety sensitivity and pain. Depress Anxiety 2010; 27:760-767.

39. Means-Christensen AJ, Roy-Byrne PP, Sherbourne CD, Craske MG, Stein MB. Relationships among pain, anxiety, and depression in primary care. Depress Anxiety 2008;25:593-600.

40. Moos RH, McCoy L, Moos BS. Global assessment of functioning (GAF) ratings: determinants and role as predictors of one-year treatment outcomes. J Clin Psychol 2000;56:449-461.

41. Söderberg P, Tungström S, Armelius BÅ. Reliability of global assessment of functioning ratings made by clinical psychiatric staff. Psychiatr Serv 2005;56:434-438.

42. Noda T, Kimura T, Sakamoto H, Seishin I. Modified electroconvulsive therapy in chronic pain. Clin Psychol 1999;41:293-295.

43. Edwards D, Gatchel R, Adams L, Stowell AW. Emotional distress and medication use in two acute pain populations: jaw and low back. Pain Pract 2006;6:242-253.

44. Barbee JG, Jamhour NJ. Lamotrigine as an augmentation agent in treatment-resistant depression. J Clin Psychiatry 2002;63:737-741.

45. Bigatti SM, Hernandez AM, Cronan TA, Rand KL. Sleep disturbances in fibromyalgia syndrome: relationship to pain and depression. Arthritis Rheum 2008;59:961-967.

46. Nicholson B, Verma S. Comorbidities in chronic neuropathic pain. Pain Med 2004;5(Suppl 1):S9-S27.

47. Bruehl S, Chung OY. Psychological and behavioral aspects of complex regional pain syndrome management. Clin J Pain 2006;22:430-437.

48. Scascighini L, Toma V, Dober-Spielmann S, Sprott H. Multidisciplinary treatment for chronic pain: a systematic review of interventions and outcomes. Rheumatology (Oxford) 2008;47:670-678.

49. Bonica JJ, Loeser JD, Chapman CR, Fordyce WE, Domenowske M. The Management of Pain. Philadelphia: Lea \& Febiger; 1990.

50. de Mos M, de Bruijn AG, Huygen FJ, Dieleman JP, Stricker BH, Sturkenboom MC. The incidence of complex regional pain syndrome: a population-based study. Pain 2007;129:12-20.

51. Schwartzman RJ, Erwin KL, Alexander GM. The natural history of complex regional pain syndrome. Clin J Pain 2009;25:273-280.

52. Sandroni P, Benrud-Larson LM, McClelland RL, Low PA. Complex regional pain syndrome type I: incidence and prevalence in Olmsted county, a population-based study. Pain 2003;103:199-207. 